\title{
Erratum to: Cervical Cancer Screening Among Southeast Asian American Women
}

\author{
Ivy K. Ho $\cdot$ Khanh T. Dinh
}

Published online: 15 September 2010

(C) Springer Science+Business Media, LLC 2010

\section{Erratum to: J Immigrant Minority Health DOI 10.1007/s10903-010-9358-0}

The original version of the online published review paper contained errors in the Introduction section of the paper and in Table 2.

While describing the socioeconomic status of Southeast Asian Americans, it was stated that "indeed, two-thirds of Southeast Asian Americans live below the poverty line [5]." The incorrect reference was given for this statement. The correct reference is listed below:

Kagawa-Singer M, Wong L, Shostak S, Walsh CR, Lew R. Breast and cervical cancer screening practices for lowincome Asian American women in ethnic-specific clinics. California Journal of Health Promotion. 2005; 3(3):180192.

Corrected portions of Table 2 are given below:
Table 2 Factors by published report and types of data analyses

\begin{tabular}{|c|c|c|}
\hline Author & Factor & $\begin{array}{l}\text { Type of analyses/ } \\
\text { OR and } 95 \% \text { CI }\end{array}$ \\
\hline \multirow[t]{6}{*}{$\begin{array}{l}\text { McPhee } \\
\text { et al. [13] }\end{array}$} & $\begin{array}{l}\text { Acculturation: No. of } \\
\text { years in US }\end{array}$ & $0.40(0.20,0.60)^{\mathrm{a}}$ \\
\hline & Marital status & $\begin{array}{l}0.10(0.07,0.20)^{\mathrm{a}} ; 0.20 \\
\quad(0.10,0.30)^{\mathrm{b}}\end{array}$ \\
\hline & Knowledge & Descriptive/qualitative \\
\hline & Financial concerns: Cost & Descriptive/qualitative \\
\hline & Access to health care & $\begin{array}{l}0.60(0.40,0.90)^{\mathrm{a}} ; 0.40 \\
\quad(0.20,0.60)^{\mathrm{b}}\end{array}$ \\
\hline & $\begin{array}{l}\text { Physician characteristics: } \\
\text { Ethnicity }\end{array}$ & $0.60(0.30,0.96)^{\mathrm{b}, \mathrm{e}}$ \\
\hline \multirow{5}{*}{$\begin{array}{l}\text { Taylor et al. } \\
{[16]}\end{array}$} & Marital status & $2.54(1.39,4.65)^{\mathrm{b}}$ \\
\hline & Financial concerns: Cost & $0.59(0.36-0.98)^{\mathrm{b}}$ \\
\hline & $\begin{array}{l}\text { Financial concerns: } \\
\text { Health insurance }\end{array}$ & Bivariate $^{\mathrm{b}}$ \\
\hline & Access to health care & $2.33(1.45-3.74)^{\mathrm{b}}$ \\
\hline & $\begin{array}{l}\text { Physician characteristics: } \\
\text { Gender }\end{array}$ & $0.53(0.33,0.88)^{\mathrm{b}, \mathrm{d}}$ \\
\hline
\end{tabular}

\footnotetext{
${ }^{\mathrm{a}}$ Ever received Pap testing; ${ }^{\mathrm{b}}$ Recently received Pap testing;

${ }^{\mathrm{d}}$ Reference group = female provider; ${ }^{\mathrm{e}}$ Reference group $=$ Non-

Vietnamese provider
}

The online version of the original article can be found under doi: 10.1007/s10903-010-9358-0.

I. K. Ho $(\bowtie) \cdot$ K. T. Dinh

Department of Psychology, University of Massachusetts Lowell, 870 Broadway Street, Lowell, MA 01854, USA

e-mail: Ivy_Ho@uml.edu 